\title{
Impact of dedicated hemostasis device for distal radial arterial access with an adequate hemostasis protocol on radial arterial observation by ultrasound
}

\author{
Yota Kawamura $^{1} \cdot$ Fuminobu Yoshimachi $^{1} \cdot$ Norihito Nakamura $^{1} \cdot$ Yoshiya Yamamoto $^{1} \cdot$ Takeaki Kudo $^{1} \cdot$ Yuji Ikari $^{2}$
}

Received: 31 October 2019 / Accepted: 17 February 2020 / Published online: 12 March 2020

(c) The Author(s) 2020

\begin{abstract}
There is no established hemostasis method or protocol for the transdistal radial approach. Therefore, this study aimed to determine whether "the PreludeSYNC DISTAL" radial compression device (PSD; Merit Medical Systems, Inc., South Jordan, UT) can effectively prevent distal radial artery (dRA) occlusion following catheterization procedures. This retrospective study analyzed patients who underwent hemostasis using the PSD from January 1, 2019, to March 31, 2019. The primary endpoint was occlusion or excessive stenosis of the radial artery (RA) 1 month after catheterization. Pulsatile blood flow and vessel diameters of the dRA and forearm RA (fRA) were measured using vascular ultrasound before and 1 month after catheterization to determine arterial damage. Secondary endpoints were achievement of hemostasis, bleeding, hematoma, aneurysm, neurological abnormality, and functional disturbance of the fingers or hand. Fifty patients (mean age, $70.9 \pm 10.7$ years; male, $72.0 \%$ ) were enrolled in this study. Complete hemostasis was achieved in all cases. Total hemostasis time was $161 \pm 45$ min. No procedure-associated complications were noted. Pulsations of the dRA and fRA were maintained at 1 month. No functional disturbance or neurological abnormality was observed. Vessel diameters of the dRA and fRA were not significantly different before and 1 month after catheterization. No dissection, pseudoaneurysm, or occlusion/stenosis was observed on ultrasound. Distal radial access with a unique device and protocol effectively achieved hemostasis and prevented injury and occlusion of the dRA and fRA.
\end{abstract}

Keywords Distal radial artery (dRA) $\cdot$ Hemostasis $\cdot$ Radial artery occlusion (RAO)

\section{Introduction}

Percutaneous coronary intervention (PCI) is an established treatment for ischemic heart disease [1-3]. A factor that affects the prognosis of PCI is the site used during the approach. The transfemoral approach (TFA) was the main approach; however, studies have indicated that the transradial approach (TRA) is more advantageous because of its fewer bleeding complications, lesser discomfort with joint mobility, lesser restriction of behavior, and better prognosis

Yota Kawamura

youta@f4.dion.ne.jp

1 Division of Cardiology, Department of Internal Medicine, Tokai University Hachioji Hospital, 1838 Ishikawa-cho Hachioji, Tokyo 192-0032, Japan

2 Division of Cardiology, Department of Internal Medicine, Tokai University School of Medicine, Isehara, Japan than TFA [4, 5]. However, radial artery (RA) occlusion (RAO) is a serious problem of TRA even in the absence of clinical symptoms. Several factors including long hemostasis time and the ratio of the RA diameter and sheath have been reported to cause RAO [6]. Therefore, sheaths with a smaller diameter and an adequate hemostasis device that can shorten the hemostasis time are necessary to effectively prevent RAO [7, 8].

Recently, a new approach involving coronary catheterization via the distal RA (dRA) at the anatomical snuffbox has been reported [9]. An advantage of this method is its shorter hemostasis time than conventional TRA [10,11]. However, no established hemostasis method or protocol for the transdistal radial approach and no suitable method of hemostasis using dRA access and a dedicated device have been reported.

A new compression hemostasis device, the PreludeSYNC DISTAL radial compression device (PSD; Merit Medical Systems, Inc., South Jordan, UT) was developed exclusively 
Fig. 1 Appearance of the PreludeSYNC DISTAL radial compression device. a The appearance of new compression device for distal radial artery: the PreludeSYNC DISTAL radial compression device (Merit Medical Systems, Inc. South Jordan, UT) is composed of a soft wristband, thumb saddle strap with loop fasteners, and inflatable balloon with an attached syringe to compress the puncture site. b Hemostasis device fixation under wrist bending. The PreludeSYNC DISTAL radial compression device is not practically misaligned despite wrist bending
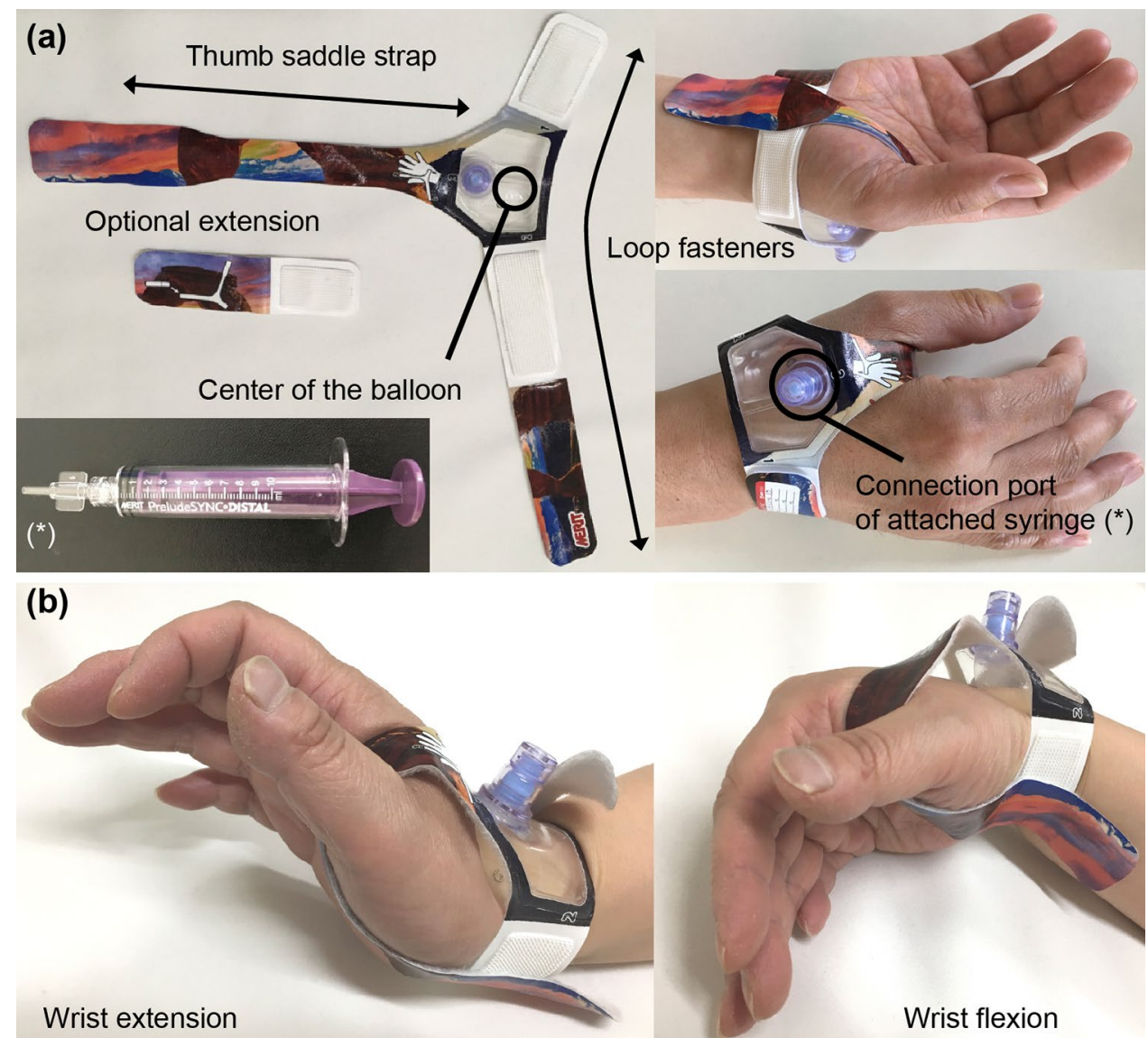

for the dRA approach (Fig. 1a). This device became available in Japan in February 2019. It is a disposable, dedicated hemostasis device that compresses the punctured dRA site with an inflatable balloon. However, the effectiveness of this device and its proper use has not been reported. To prevent injury and occlusion of the dRA and forearm RA (fRA), a new protocol was created at our hospital and performed during hemostasis of the dRA.

During this study, the effectiveness of the PSD and our protocol for preventing dRA or fRA injury and occlusion was researched retrospectively.

\section{Methods}

\section{Case selection}

This was a sequential retrospective observational study performed at a single center. Patients who underwent coronary catheterization via the dRA for the first time and with the use of the PSD as a hemostasis device from January 1, 2019, to March 31, 2019 were enrolled (Fig. 2). Patients underwent diagnostic coronary angiography (CAG) or PCI according to the usual indications. Moreover, patients underwent cannulation via the radial or distal radial approach, except those on dialysis or requiring dialysis in the near future. Arterial diameters of the fRA and dRA before the coronary catheterization procedure were measured using vascular ultrasound. Cross-sectional diameters of the fRA and dRA at the point of puncture were measured, and the dRA approach with a $5 \mathrm{Fr}$ sheath of a small diameter or a $4 \mathrm{Fr}$ conventional sheath was selected only for patients with dRA diameters of $>2.0 \mathrm{~mm}$ to avoid injury from the large sheath. If a $6 \mathrm{Fr}$ sheath is needed because of the strategy, then the diameter of the artery would needed to be $>2.5 \mathrm{~mm}$. Exclusion criteria were acute coronary syndrome requiring emergent PCI, failed dRA puncture, sheath insertion from the dRA, and difficulty scheduling follow-up visits.

All patients provided informed written consent, and the study conformed to institutional ethics guidelines and those of the American Physiological Society.

\section{Coronary catheterization procedure}

After the administration of local anesthesia, a disposable plastic-cannulated needle was inserted in the dRA with pulse palpable or vascular ultrasound using the Seldinger technique, and a hydrophilic-coated wire attached to the sheath system was inserted carefully. A sheath with an adequate size for the procedure was inserted. A $30 \mathrm{~cm} 4$ Fr sheath 
Fig. 2 Hemostasis protocol with the PreludeSYNC DISTAL radial compression device and observational procedure

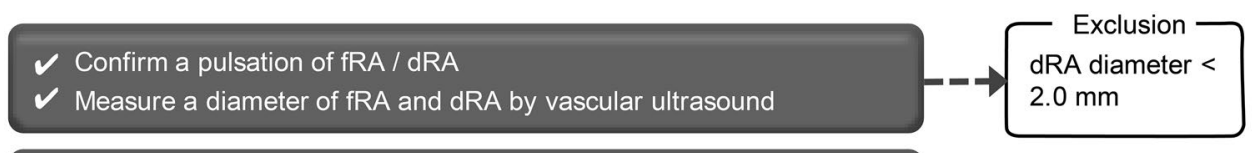

Elective CAG (4Fr.) / PCI (5Fr. or 6Fr. Glidesheath Slender) procedure via $1^{\text {st }}$ time $d R A$ successfully

Sheath removal after ACT measurement and hemostasis with the PreludeSYNC DISTAL Radial (10 $\mathrm{ml}$ air inflation by attached syringe)

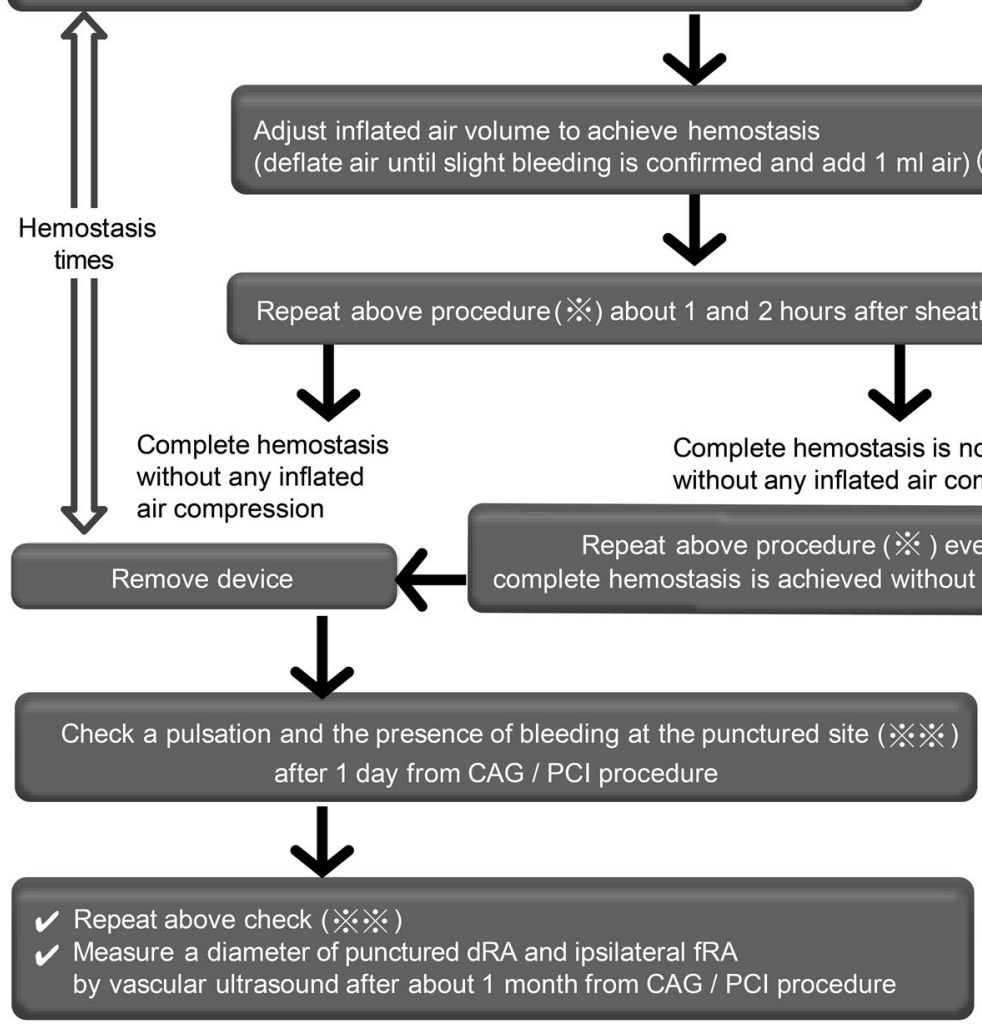

(Supersheath, Medikit, Tokyo, Japan) was used for CAG and a $16 \mathrm{~cm} 5$ Fr or 6 Fr sheath [Glide Sheath Slender (GSS); Terumo, Tokyo, Japan] [12] for PCI. Unfractionated heparin was administered intra-arterially via the sheath. The initial injected dose of unfractionated heparin was 2000 units for CAG and 5000 units for PCI. Additional 2000 units of unfractionated heparin were administered every hour. After finishing the procedures, the activated clotting time (ACT) was measured before sheath removal. These procedures were performed by various physicians with experience performing coronary catheterization procedures via the dRA.

\section{Hemostasis of the dRA after CAG or PCl using the PSD}

The PSD for hemostasis was used for dRA hemostasis after coronary catheterization as follows:

1. Clean and dry the dRA site with ethanol.
2. Prepare the PSD for the right hand or left hand as appropriate.

3. Withdraw the sheath at approximately 1 inch.

4. Place the PSD using the black spot as the landmark at the center of the balloon to access the puncture site.

5. Tightly fasten the band around the wrist.

6. Wrap band between the thumb and forefinger without slack.

7. Fill the attached exclusive syringe with $10 \mathrm{~mL}$ of air and connect the valve on top of the device.

8. Slowly inflate the balloon with air while simultaneously removing the sheath. When the sheath is completely removed, continue injecting air in the balloon until hemostasis is complete.

9. Adjust the inflated air volume to achieve hemostasis (deflate air until slight bleeding is confirmed and add $1 \mathrm{~mL}$ of air using the syringe).

10. Disconnect the syringe.

11. Approximately $1 \mathrm{~h}$ after sheath removal, adjust the inflated air volume to achieve hemostasis as in step 9. 
12. Approximately $2 \mathrm{~h}$ after sheath removal, adjust the inflated air volume to achieve hemostasis as in step 9.

13. Remove the PSD if complete hemostasis is achieved without inflated air compression. If complete hemostasis is not achieved, then repeat step 9 every hour until complete hemostasis is achieved.

14. Record the time and inflated air volume of each process.

15. During the hemostasis period, there is no restriction of wrist movement (Fig. 1b).

\section{Follow-up of the punctured dRA site}

Pulsation and the presence of complications associated with hemostasis procedure (detailed below) were checked after 1 day and approximately 1 month after the procedure. At 1 month follow-up examination, patency of the artery and arterial diameters of the fRA and dRA were measured by vascular ultrasound using the same method prior to coronary catheterization.

\section{Endpoints}

The primary endpoint was occlusion or excessive stenosis of the RA at 1 month after catheterization. Pulsatile blood flow and the vessel diameters of the dRA and fRA using vascular ultrasound were examined. Arterial diameters of the fRA and IRA were compared with those before coronary catheterization using Student's $t$ test and SPSS software version 25 (IBM Corp., Armonk, NY).

Secondary endpoints were as follows: successful and safe hemostasis at the dRA puncture site; hemostasis time; vascular complications associated with the procedure, including bleeding, hematoma and pseudoaneurysm; neurological abnormality; and functional disturbances of the fingers or hand.

Hemostasis time was defined as the period from sheath removal to complete detachment of the PSD. Bleeding events were defined according to the BARC criteria [13]. A minor hematoma was defined as $<3.0 \mathrm{~cm}$ without symptoms, and a major hematoma was defined as $>3.0 \mathrm{~cm}$ or the presence of symptoms caused by the hematoma. The existence of a pseudoaneurysm was checked by ultrasound at approximately 1 month after the procedure. Neurological sequelae were defined as symptomatic abnormal feelings associated with the hemostasis procedure. Functional disturbance was defined as paralysis or disorders of hand and/or finger movements caused by the hemostasis procedure.

\section{Results}

Fifty patients were enrolled consecutively during this study. Background characteristics and coronary catheterization procedures are shown in Table 1 . The mean age was
Table 1 Background of patients and coronary catheterization procedures

\begin{tabular}{|c|c|}
\hline Number of cases & 50 \\
\hline Mean age (years) & $70.9 \pm 10.7$ \\
\hline Male sex $(\%)$ & $36(72.0 \%)$ \\
\hline Body mass index $\left(\mathrm{kg} / \mathrm{m}^{2}\right)$ & $24.1 \pm 4.1$ \\
\hline \multicolumn{2}{|l|}{ Prevalence of coronary risk factor } \\
\hline Hypertension & $35(70.0 \%)$ \\
\hline Dyslipidemia & $39(78.0 \%)$ \\
\hline Diabetes & $22(44.0 \%)$ \\
\hline Current smoker & $23(46.0 \%)$ \\
\hline Chronic kidney disease & $4(8.0 \%)$ \\
\hline \multicolumn{2}{|l|}{ Antiplatelet therapy } \\
\hline Aspirin & $38(76.0 \%)$ \\
\hline Clopidogrel & $22(44.0 \%)$ \\
\hline Prasugrel & $9(18.0 \%)$ \\
\hline Dual antiplatelet therapy & $21(42.0 \%)$ \\
\hline \multicolumn{2}{|l|}{ Anticoagulant therapy } \\
\hline Warfarin & $3(6.0 \%)$ \\
\hline Direct oral anticoagulants & $6(12.0 \%)$ \\
\hline Dual antiplatelet plus anticoagulant therapy & $5(10.0 \%)$ \\
\hline \multicolumn{2}{|l|}{ Coronary catheterization } \\
\hline Coronary angiography & $32(64.0 \%)$ \\
\hline Coronary intervention & $18(36.0 \%)$ \\
\hline \multicolumn{2}{|l|}{ Sheath size $(\%)$} \\
\hline $4-\mathrm{F}$ & $31(62.0 \%)$ \\
\hline 5-F GSS & $16(32.0 \%)$ \\
\hline 6-F GSS & $3(6.0 \%)$ \\
\hline Heparin administration (U) & $3340.0 \pm 1901.8$ \\
\hline Activated clotting time at sheath removal (s.) & $252.9 \pm 54.1$ \\
\hline Inflated air volume after sheath removal (mL) & $8.2 \pm 1.3$ \\
\hline
\end{tabular}

GSS glidesheath slender

$70.9 \pm 10.7$ years, and $72.0 \%$ patients were men. Thirty two patients underwent CAG (4 Fr sheath for 31 patients; $5 \mathrm{Fr}$ sheath for 1 patient). PCI was performed for 18 patients (15 with the 5 Fr GSS and 3 with the 6 Fr GSS). Each dRA diameter of patients who underwent PCI with the 6 Fr GSS was $>3.0 \mathrm{~mm}$. Twenty-one patients received dual antiplatelet therapy (DAPT). Nine patients received anticoagulant therapy, including five patients who received both DAPT and anticoagulant therapy. The mean adjusted inflated air volume after sheath removal at the start of hemostasis was $8.2 \pm 1.3 \mathrm{~mL}$.

The mean duration of hemostasis time was $161 \pm 45 \mathrm{~min}$ (Table 2). There were no complications associated with the hemostasis procedure. No occlusions of the dRA were observed after removing the PSD or 1 day after the procedure, which was the day of discharge.

No occlusions, stenoses, pseudoaneurysms, or dissections of the dRA and fRA were observed on ultrasound approximately 1 month after catheterization. No functional 
Table 2 Hemostasis and complications on the day after catheterization

\begin{tabular}{ll}
\hline $\begin{array}{l}\text { Duration of hemostasis (min) } \\
\text { dRA pulsation (\%) }\end{array}$ & $161 \pm 45$ \\
fRA pulsation (\%) & $50(100 \%)$ \\
Complication related to hemostasis procedure (\%) & $50(100 \%)$ \\
Bleeding (any type of BARC definition) & $0(0 \%)$ \\
Major hematoma & $0(0 \%)$ \\
Minor hematoma & $0(0 \%)$ \\
Pseudoaneurysm & $0(0 \%)$ \\
Neurological sequela & $0(0 \%)$ \\
\hline
\end{tabular}

$B A R C$ Bleeding Academic Research Consortium, $d R A$ distal radial artery, $f R A$ forearm radial artery

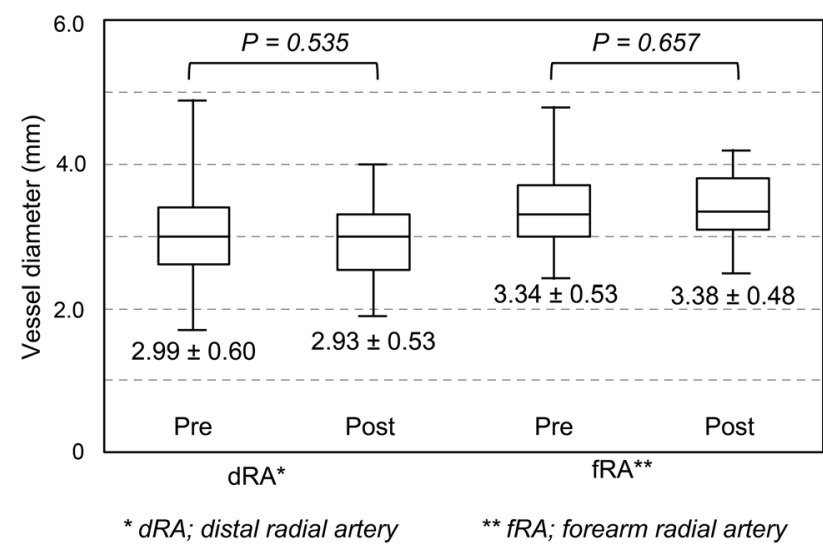

Fig. 3 Diameter of the distal radial artery and forearm radial artery compared before and after catheterization

disturbance was observed. Vessel diameters of the $\mathrm{dRA}$ and fRA before and after catheterization were compared, and no significant differences were observed between these measurements (Fig. 3).

\section{Discussion}

This is the first report of the dRA condition observed using ultrasound and a patent hemostasis protocol with a dedicated hemostasis device after the transdistal radial approach to coronary catheterization. Using the PSD and a simple hemostatic protocol, blood flow in both the dRA and fRA without damage, as observed using ultrasound was maintained 1 month after catheterization and complete hemostasis without complications was achieved in all cases.

Conventional TRA has been the standard method because of its many advantages including lower rates of local vascular complications such as hematoma and pseudoaneurysm, less bleeding, better comfort, lower costs, shorter hospital stays, and decreased workload for nursing staff
[14]. Nevertheless, TRA also has some serious and unresolved problems, including the limitation of the sheath size and RAO, which limits the fRA as a future access site and disturbs creating the shunt for hemodialysis. The reported occlusion rate of the fRA after TRA is 4-12\% [15-17]. The main causes are fRAs with a small diameter and the hemostasis method.

However, the transdistal radial approach for coronary catheterization has a relatively shorter history than the TRA. Therefore, many issues associated with the transdistal radial approach have not yet been resolved. Generally, the dRA is smaller than the fRA and has many anatomical variations because of its peripheral location. Transradial interventions became safer after studying hemostatic devices and their proper use. Therefore, to further pursue the benefits of using the dRA and to avoid complications of dRA injuries and occlusions, a slender catheter and an adequate hemostasis method with a dedicated hemostasis device are needed to improve the distal radial approach.

The long history of the conventional radial approach has given insights into how to avoid damage to the artery, it is necessary to use a sheath smaller than the vessel diameter to achieve a secure puncture and gradually decompress and release the hemostasis device early to avoid RAO.

Before the launch of the PSD as the first dedicated hemostasis device for the distal radial approach, various hemostasis methods involving elastic tape and bandages have been suggested. These techniques had a learning curve and it was impossible to calculate the fixing power of the bandages for each patient. Furthermore, their uniqueness has made it difficult to establish standard hemostasis protocols. In contrast, the PSD is simple to use with a uniform protocol for dRA hemostasis.

During this study, no bleeding complications were observed, and blood flow in the dRA was maintained at 1 month after the procedure in all cases. These results demonstrate that our hemostatic protocol with pressure reduction of the PSD to the greatest extent possible and more than once effectively avoided arterial occlusion.

Excluding cases with $\mathrm{dRA}$ of diameters $<2.0 \mathrm{~mm}$ may have affected the results of this study. The sensitivity and specificity for detecting severe flow reduction of the fRA versus the ratio of the fRA diameter to the sheath outer diameter were between 1.0 and 1.1 in the previous study, respectively $[6,18]$. Therefore, a threshold of $2.0 \mathrm{~mm}$ was determined to be the threshold diameter for the distal radial approach using the 5 Fr GSS [12]. Of course, if the dRA and fRA diameters are larger than the outer diameter of the sheath, then a sheath with a larger diameter could be inserted. However, using a sheath with a larger diameter may increase the hemostasis time, as has been experienced with conventional transradial or transfemoral intervention, and this may cause bleeding complications or occlusion of 
the artery. Therefore, adequate case selection in accordance with our protocol and the selection of an appropriate sheath size may have contributed to the good outcomes obtained in our study.

The ultrasound, which is a non-invasive examination modality, was used at all catheterization sites to measure the vessel diameter and to observe the condition of the artery. We believe that cannulation of a vessel without measuring the size or recognizing the location of the vessel should not be performed. Ultrasound-guided puncture is recommended and considered an excellent step when using the conventional radial, femoral, and distal radial approaches [19-22]. The fRA is an access site in hemodialysis patients that should be avoided because RAO, which is a serious problem, can occur when creating vascular access. However, the transdistal radial approach for coronary catheterization and our hemostasis protocol may be useful for hemodialysis and avoid fRA and dRA occlusion. Because the dRA occlusion ratio may be low and fRA is located proximal site of the bifurcation into the $\mathrm{dRA}$ and ramus volaris superficialis, fRA may not be occluded.

The PSD hemostasis device has many features. The transparent balloon may contribute to the early detection of recurrent bleeding by nurses or patients. Its soft wristband and thumb saddle strap also contribute to secure hemostasis. The puncture site of the dRA is located in the snuffbox near the carpometacarpal joint of the thumb. Previously, with classical bandage hemostasis after sheath removal, movement of the carpometacarpal joint caused misalignment of the puncture point and hemostasis device, possibly preventing stable pressurization. Furthermore, movement of the carpometacarpal joint, including palmar abduction and adduction, radial abduction, and ulnar adduction, might also cause misalignment of the bandage. Compared with other balloon inflation type devices, the PSD has the advantage of being dedicated to the dRA. Its most remarkable characteristic is the thumb saddle strap that prevents longitudinal misalignment that can occur with various movements of the carpometacarpal joint.

No patients in this study had recurrent bleeding from the puncture site during hemostasis with the PSD despite unrestricted wrist movement during the hemostasis period (Fig. 1b). This suggests that hemostasis of the punctured dRA site using the PSD is more comfortable for patients than any classical method for the dRA or other conventional TRA that dose not allow patients to move the wrist during hemostasis.

One of the disadvantages of the PSD is its cost. Hemostasis with a simple bandage is less expensive than using a new exclusive device. The cost-effectiveness of each device must be considered in the context of the increasing costs of healthcare.

\section{Study limitations}

This study had several limitations. First, it was a singlecenter retrospective study that included a small number of patients. Second, the hemostasis protocol and hemostasis device were not compared with others. Third, the specific sheath had a limited size of $<6 \mathrm{Fr}$, and was used during daily angiography and PCI. A multicenter, prospective registry including the use of larger sheaths for coronary catheterization and comparing this hemostasis protocol with other protocols and hemostasis devices is expected to demonstrate the superiority of our hemostasis protocol. Fourth, patients with dRA diameters of the dRA $<2.0 \mathrm{~mm}$ were excluded. If this threshold was changed, then different results might be obtained. Using this threshold, the distal radial approach was performed for approximately 50\% of the total transradial interventions during our daily practice. This must be revised to achieve the benefits of dRA in more patients. Fifth, the arterial diameter was measured during vascular ultrasound examinations by several physicians in this study. Therefore, measurement mismatches were possible to due to subjectivity.

\section{Conclusion}

An adequate hemostatic protocol with the PSD led to blood flow maintenance in both the dRA and fRA without damage at 1 month after catheterization. Complete hemostasis without complications was achieved in all cases. If the issue of cost is solved, then patent hemostasis methods using the PSD could become the standard procedure for puncturing dRA sites.

Funding This study was not funded by any company.

\section{Compliance with ethical standards}

Conflict of interest The authors have no conflicts of interest directly relevant to the content of this article.

Ethical approval This study had been approved by the research ethics committee of Tokai University Hachioji Hospital.

Informed consent Written informed consent was obtained from all patients in this study.

Open Access This article is licensed under a Creative Commons Attribution 4.0 International License, which permits use, sharing, adaptation, distribution and reproduction in any medium or format, as long as you give appropriate credit to the original author(s) and the source, provide a link to the Creative Commons licence, and indicate if changes were made. The images or other third party material in this article are 
included in the article's Creative Commons licence, unless indicated otherwise in a credit line to the material. If material is not included in the article's Creative Commons licence and your intended use is not permitted by statutory regulation or exceeds the permitted use, you will need to obtain permission directly from the copyright holder. To view a copy of this licence, visit http://creativecommons.org/licenses/by/4.0/.

\section{References}

1. Andersen HR, Nielsen TT, Rasmussen K, et al. A comparison of coronary angioplasty with fibrinolytic therapy in acute myocardial infarction. N Engl J Med. 2003;349:733-42.

2. Grines CL, Westerhausen DR, Grines LL, et al. A randomized trial of transfer for primary angioplasty versus on-site thrombolysis in patients with high-risk myocardial infarction: the Air Primary Angioplasty in Myocardial Infarction study. J Am Coll Cardiol. 2002;39:1713-9.

3. Widimský P, Groch L, Zelizko M, Aschermann M, Bednář F, Suryapranata H. Multicentre randomized trial comparing transport to primary angioplasty vs immediate thrombolysis vs combined strategy for patients with acute myocardial infarction presenting to a community hospital without a catheterization laboratory. The PRAGUE study. Eur Heart J. 2000;21:823-31.

4. Jolly SS, Yusuf S, Cairns J, et al. Radial versus femoral access for coronary angiography and intervention in patients with acute coronary syndromes (RIVAL): a randomised, parallel group, multicentre trial. Lancet. 2011;377(9775):1409-20.

5. Valgimigli M, Gagnor A, Calabró P, et al. Radial versus femoral access in patients with acute coronary syndromes undergoing invasive management: a randomised multicentre trial. Lancet. 2015;385:2465-76

6. Saito $\mathrm{S}$, Ikei H, Hosokawa G, Tanaka S. Influence of the ratio between radial artery inner diameter and sheath outer diameter on radial artery flow after transradial coronary intervention. Catheter Cardiovasc Interv. 1999;46:173-8.

7. Rathore S, Stables RH, Pauriah M, et al. A randomized comparison of TR band and radistop hemostatic compression devices after transradial coronary intervention. Catheter Cardiovasc Interv. 2010;76:660-7.

8. Pancholy S, Coppola J, Patel T, Roke Thomas M. Prevention of radial artery occlusion-patent hemostasis evaluation trial (PROPHET study): a randomized comparison of traditional versus patency documented hemostasis after transradial catheterization. Catheter Cardiovasc Interv. 2008;72:335-40.

9. Kiemeneij F. Left distal transradial access in the anatomical snuffbox for coronary angiography (ldTRA) and interventions (ldTRI). EuroIntervention. 2017;13(7):851-7.

10. Aoi $\mathrm{S}, \mathrm{Htun} \mathrm{WW}$, Freeo S, et al. Distal transradial artery access in the anatomical snuffbox for coronary angiography as an alternative access site for faster hemostasis. Catheter Cardiovasc Interv. 2019;94(5):651-7.

11. Aminian A, Saito S, Takahashi A. Impact of sheath size and hemostasis time on radial artery patency after transradial coronary angiography and intervention in Japanese and non-Japanese patients: a substudy from RAP and BEAT (Radial Artery Patency and Bleeding, Efficacy, Adverse evenT) randomized multicenter trial. Catheter Cardiovasc Interv. 2018;92(5):844-51.

12. Yoshimachi F, Kiemeneij F, Masutani M, Matsukage T, Takahashi A, Ikari Y. Safety and feasibility of the new 5 Fr Glidesheath Slender. Cardiovasc Interv Ther. 2016;31:38-41.

13. Mehran R, Rao SV, Bhatt DL, et al. Standardized bleeding definitions for cardiovascular clinical trials: a consensus report from the Bleeding Academic Research Consortium. Circulation. 2011;123:2736-47.

14. Cohen MG, Ohman EM. Should the benefit of transradial access still be questioned? JACC Cardiovasc Interv. 2016;9:908-10.

15. Takeshita $\mathrm{S}$, Asano $\mathrm{H}$, Hata $\mathrm{T}$, et al. Comparison of frequency of radial artery occlusion after 4 Fr versus 6 Fr transradial coronary intervention (from the Novel Angioplasty USIng Coronary Accessor Trial). Am J Cardiol. 2014;113:1986-9.

16. Petroglou D, Didagelos M, Chalikias G, et al. Manual versus mechanical compression of the radial artery after transradial coronary angiography: the MEMORY multicenter randomized trial. JACC Cardiovasc Interv. 2018;11:1050-8.

17. Cubero JM, Lombardo J, Pedrosa C, Diaz D, et al. Radial compression guided by mean artery pressure versus standard compression with a pneumatic device (RACOMAP). Catheter Cardiovasc Interv. 2009;73:467-72.

18. Naito T, Sawaoka T, Sasaki K, Iida K, Sakuraba S, Yokohama K, Sato H, Soma Okamura E, Harada T, Yoshimachi F. Evaluation of the diameter of the distal radial artery at the anatomical snuff box using ultrasound in Japanese patients. Cardiovasc Interv Ther. 2019;34(4):312-6.

19. Slattery MM, Goh GS, Power S, Given MF, McGrath FP, Lee MJ. Comparison of ultrasound-guided and fluoroscopy-assisted antegrade common femoral artery puncture techniques. Cardiovasc Interv Radiol. 2015;38:579-82.

20. Gopalasingam N, Hansen MA, Thorn S, Sloth E, Juhl-Olsen P. Ultrasound-guided radial artery catheterisation increases the success rate among anaesthesiology residents: a randomised study. J Vasc Access. 2017;18:546-51.

21. Sobolev M, Slovut DP, Lee Chang A, Shiloh AL, Eisen LA. Ultrasound-Guided catheterization of the femoral artery: a systematic review and meta-analysis of randomized controlled trials. J Invasive Cardiol. 2015;27(7):318-23.

22. Gao YB, Yan JH, Gao FQ, Pan L, Wang XZ, Lv CJ. Effects of ultrasound-guided radial artery catheterization: an updated metaanalysis. Am J Emerg Med. 2015;33(1):50-5.

Publisher's Note Springer Nature remains neutral with regard to jurisdictional claims in published maps and institutional affiliations. 